\title{
Improved real-time control on the Apple by mimicking an on-board $60-\mathrm{Hz}$ clock
}

\author{
JOHN O. MITTERER \\ Brock University, St. Catharines, Ontario L2S 3A1, Canada \\ and \\ BRUCE OSBORNE \\ Trent University, Peterborough, Ontario K9J 7B8, Canada
}

\begin{abstract}
By delivering the vertical synch pulse to the maskable interrupt, it is possible to supply the Apple with a functional on-board $60-\mathrm{Hz}$ clock that operates virtually independently of the CPU. This modification makes it easier to program more complex experiments involving independent control over stimulus exposure duration and the timing of response latency.
\end{abstract}

The use of the Apple as a substitute for a tachistoscope is by now well established. Using hardware modifications first suggested by Reed (1979) and stimulus display methods discussed by Cavanagh and Anstis (1980), it is possible to gain precise control over multiple displays, each with durations in multiples of $16.67 \mathrm{msec}$. This can be coupled with millisecond-accurate response timing (e.g., Price, 1979) to allow many experiments to be done quite easily. For example, experiments involving a response-terminated display need to sense the vertical synch pulse once to synchronize display and response timing onset. Then the response can be sensed to terminate the display and the software timer. Similarly, displaying a stimulus for a fixed interval involves sensing the vertical synch pulse to initiate the display and then counting the required number of pulses before terminating the display.

This technology becomes more difficult to use when more complex designs are required, such as displaying stimuli for brief durations while simultaneously timing response latencies. One way to do this is to use a routine to time the stimulus display and then to start a response timing routine that is terminated by the response. Reaction time is then calculated by adding the elapsed time on the timer to the length of time the display was on (in increments of $16.67 \mathrm{msec}$ ). While this can be done, problems can arise if, for example, a response can sometimes occur before stimulus display is completed. In this case, reaction time would be incorrectly set to display duration. While this problem may be surmounted with a two-stage timing routine that uses the same memory locations for the display timing count

Requests for reprints and information should be addressed to John O. Mitterer, Department of Psychology, Brock University, St. Catharines, Ontario L2S $3 \mathrm{~A} 1$, Canada. The development of this equipment was supported by Natural Sciences and Engineering Research Council of Canada Grant A8304 to the first author. and the response timing count, the software involved can become complex. In general, any time both display timing (using the vertical synch pulse) and response timing (to millisecond accuracy) must occur independently and concurrently, CPU timesharing problems of greater or lesser magnitude arise. An alternative solution is to use hardware to control these timing functions. This can be done easily on any system (such as the Commodore PET) that has an on-board $60-\mathrm{Hz}$ clock, but not on the Apple. Fortunately, a simple modification of a suggestion originally put forward by Reed (1979) allows the Apple to be supplied with such a capability.

Reed (1979) suggested that the vertical synch pulse be delivered to one of the three digital sense switches available to the user. Rather than do this, the vertical synch pulse can be delivered to the maskable interrupt (IRQ), which can be accessed at Pin $\mathbf{3 0}$ of any available expansion slot. The easiest way to do this is to make up a board that will sit in the desired slot. The vertical synch pulse can then be routed to this board, where it can be appropriately shaped and connected to Pin 30 . In addition, it is useful to mount a switch on the card that allows the vertical synch pulse to be turned off at will.

It is useful to deliver the vertical synch pulse to the IRQ rather than to the nonmaskable interrupt (NMI) to avoid having an in terrupt generated every $16.67 \mathrm{msec}$ regardless of anything else (for a good discussion of interrupt processing on the Apple, see White, 1981). Also, it is useful to have the hardware switch available so that the vertical synch pulse circuit can easily be totally disabled when it is not required. While a "cold start" of the Apple sets the 6502's interrupt disable (I) flag automatically, it is good practice to use the hardware switch to turn the vertical synch pulse circuit on only when the program requiring it has been run and to turn it off before using any other software. 
This modification can be implemented in several ways. The vertical synch pulse can be picked up at several pins on the D11 chip. Pin 12 gives an output of $60 \mathrm{~Hz}$ composed of $8.1 \mathrm{msec}$ off followed by $8.56 \mathrm{msec}$ on, Pin 15 gives 60 positive-going 1 -microsec pulses per second, and Pin 9 gives 60 negative-going 1-microsec pulses per second. The IRQ requires a negative pulse level for about 6.5 microsec for reliable operation. Any scheme that picks up the vertical synch pulse at any of these points and shapes it appropriately for delivery to the IRQ will do the job. Our implementation uses the pulse at Pin 9 on Chip D11, which is delivered to a board mounted in Apple expansion Slot 7. That 1-microsec pulse drives a 7-microsec one-shot whose output is routed through a hardware switch before being delivered to Pin 30 on the expansion slot. Finally, we have taken power of $+5 \mathrm{dc}$ from Pin 14 of the D11 chip to drive the interrupt. This power can come from many different sources.

With this hardware modification, it is easy to use the interrupt servicing routine to count vertical synch pulses when desired. Such a routine requires only a few machine cycles to execute and, hence, takes little time away from the primary task to which the CPU is devoted. The Apple itself requires about 54 cycles to get to and from the interrupt servicing routine, and the simplest routine takes only 5 or 10 cycles. To calculate the time for servicing one interrupt, it is only necessary to count the number of machine cycles involved. Since each machine cycle is $9.7779 \mathrm{E}-07 \mathrm{sec}$ in duration (Price, 1979), it is easy to correct the main timing results accordingly. For example, the routine we use requires 80 cycles for each interrupt. If we were displaying the stimulus for a full second, then our reaction timing result would have to be corrected by $60 * 80 * 9.7779 \mathrm{E}-07 \mathrm{sec}$ to be accurate. Functionally, such a routine behaves like an independent on-board $60-\mathrm{Hz}$ clock that can be disabled or enabled simply by setting or not setting the 6502's I flag.
A word of warning is in order for anyone wishing to implement this (or any similar) modification: Empirically verify every step. In particular, the accuracy of the resulting display and response timing routines should be checked before use. For example, we have found that the interpulse interval of the vertical synch pulse varies from machine to machine by as much as $.67 \mathrm{msec}$. Also, some machines produce vertical synch pulses (particularly at Pin 9 on the D11 chip) contaminated by spurious activity also capable of generating interrupts. Both of these problems can seriously affect the accuracy of timing routines.

With this modification, it is possible to have the interrupt servicing routine control stimulus exposure durations while the $\mathrm{CPU}$ is busy timing response latencies. Of course, any other application requiring an independent $60 \cdot \mathrm{Hz}$ clock can also benefit. For example, the interrupt-driven clock can be used to time response latency, providing that $16.67-\mathrm{msec}$ accuracy is sufficient for the purpose at hand. To do this, the response pulse can be delivered to the NMI. Thus response timing can take place without much CPU overhead. This is desirable if, for example, dynamic updating of screen displays is required independently of response latency timing.

\section{REFERENCES}

Cavanagh, P., \& Anstis, S. M. Visual psychophysics on the Apple II: Getting started. Behavior Research Methods \& Instrumentation, 1980, 12, 614-626.

Paice, J. M. Software timing for 6500 series microcomputers. Behavior Research Methods \& Instrumentation, 1979, 11, 568571.

REed, A. V. Microcomputer display timing: Problems and solutions. Behavior Research Methods \& Instrumentation, 1979, 11, 572-576.

WhITE, G. M. Using interrupts on the APPLE II system. BYTE, 1981, 6(5), 280-294.

(Received for publication March 22, 1982; revision accepted June 4, 1982.) 